Revista Brasileira de Agricultura Irrigada v.10, nº.3, p. 621 - 630, 2016

ISSN 1982-7679 (On-line)

Fortaleza, CE, INOVAGRI - http://www.inovagri.org.br

DOI: $10.7127 /$ rbai.v10n300379

Protocolo 379.16 - 25/11/2015 Aprovado em 15/06/2016

\title{
ESTIMATIVA DA EVAPOTRANSPIRAÇÃO DE REFERÊNCIA POR DIFERENTES MÉTODOS PARA O MUNICÍPIO DE SÃO LUIS - MA
}

\author{
Christian José Mendoza ${ }^{1}$, Ronaldo Harold Menezes ${ }^{2}$, Antonio Solon Dias ${ }^{3}$
}

\begin{abstract}
RESUMO
A Estimativa da evapotranspiração é de grande importância na modelagem hidrológica e no manejo de bacias hidrográficas como gestão dos recursos hídricos de uma região sendo indispensável no manejo hídrico sustentável de cultivos irrigados. Neste sentido, o presente trabalho tem por objetivo avaliar o desempenho dos métodos Blaney-Criddle, Camargo, Hargreaves-Samani e Priestley-Taylor, de estimativa da evapotranspiração de referência em base diária, para a região de São Luis, MA, com base em comparações com o método padrão Penman-Monteith. Foram analisados dados coletados a partir de uma estação automática do INMET localizada em São Luis - MA, sob coordenadas geográficas de $2^{\circ} 31^{\prime} 48^{\prime \prime}$ de latitude sul, 44¹2’36” de longitude oeste Greenwich e altitude aproximada de 50,86 m. Conforme classificação de Köppen, o clima da região é do tipo “Aw”. Foram quantificados valores diários das temperaturas máxima (Tmáx), média (Tméd) e mínima (Tmín); umidade relativa média do ar (URméd.), insolação (n) e velocidade do vento a 10m (Vv). Os dados foram obtidos de 01 de janeiro de 2012 a 30 de dezembro de 2012. O processamento dos dados foi realizado através do uso do editor de planilhas do software Microsoft@ Office Excel ${ }^{\circledR}$, onde foram calculadas as diárias de evapotranspiração (ETo). Os valores de $\mathrm{ET}_{\mathrm{O}}$ variam de 1,95 mm a 5,79 mm, no método padrão Penman-Monteith. Com apoio no erro absoluto médio (EAM), verificou-se, dentre outros indicadores, que o melhor desempenho foi obtido com o método de PriestleyTaylor. Em relação aos métodos aplicáveis quando apenas dados de temperatura foram disponíveis, o de Hargreaves-Samani foi o que apresentou melhor desempenho com c = 0,64.
\end{abstract}

Palavras-Chave: Evapotranspiração, Erro absoluto médio, Estimativa.

\section{ESTIMATE OF REFERENCE EVAPOTRANSPIRATION FOR DIFFERENT METHODS IN THE CITY SÃO LUIS, MARANHÃO STATE, BRAZIL}

\author{
ABSTRACT \\ 1 Prof. Doutor, Departamento de Engenharia Agrícola, CCA/UEMA, São Luis, MA, \\ christianmendozac@gmail.com \\ ${ }^{2}$ Prof. Adjunto, Departamento de Engenharia Agrícola, CCA/UEMA, São Luis, MA, rhmenezes@yahoo.com.br \\ 3 Prof. Adjunto II, Departamento de Engenharia Agrícola, CCA/UEMA, São Luis, MA, solon1948- \\ sl@hotmail.com
}


The estimation of evapotranspiration is of great importance in hydrologic modeling and watershed management and water resources management of a region being essential to sustainable water management of irrigated crops. In this sense, the present paper aims to evaluate the performance of the Blaney-Criddle, Camargo, Samani-Hargreaves and PriestleyTaylor methods, estimation of reference evapotranspiration on a daily basis, for the region of Sao Luis, MA, based on comparisons with the standard Penman-Monteith method. Data collected were analyzed from an automatic station of INMET located in San Luis - MA, in geographic coordinates $2^{\circ} 31^{\prime} 48$ "south latitude, $44^{\circ} 12^{\prime} 36^{\prime \prime}$ west longitude Greenwich and approximate altitude of $50.86 \mathrm{~m}$. According to Köppen classification, the climate is Aw. Daily values of maximum temperatures (Tmax), mean temperature (Tméd) and minimum temperature (Tmin); average relative humidity (URméd.), sunshine (n) and wind speed at $10 \mathrm{~m}(\mathrm{Vv})$ were quantified. Data were collected from January 1, 2012 to December 30, 2012. Data processing was performed by using a spreadsheet in Microsoft ${ }^{\circledR}$ Office Excel ${ }^{\circledR}$ software, which was calculated from the daily evapotranspiration. ETo values ranging from $1.95 \mathrm{~mm}$ to $5.79 \mathrm{~mm}$, in standard Penman-Monteith method. With support in the mean absolute error (MAE), it was found, among other indicators, the best performance was obtained with the method of PriestleyTaylor. Regarding the methods applicable when only temperature data were available, the Hargreaves-Samani showed the best performance.

Keywords: Evapotranspiration, mean absolute error, estimate

\section{INTRODUÇÃO}

O conhecimento da evapotranspiração de referencia - ETo é de fundamental importância em atividades ligadas a gestão de bacias hidrográficas, em modelagens meteorológica e hidrológica e, sobretudo, no manejo hídrico da agricultura irrigada (Ferraz, R. C., 2014).

Muitos trabalhos utilizam método empíricos de estimativa da ETo. Entretanto, a não verificação da adequação dos métodos de estimativa da evapotranspiração de referência às condições climáticas locais, a falta de precisão na estimativa, bem como o erro, devido ao uso de instrumentos de medidas inadequados, em geral, também conduzem ao manejo inadequado da água, afetando muitas vezes a produção agrícola. Aplicações de água insuficientes ou em excesso resultam em perdas e prejuízos consideráveis às plantas e ao solo, diminuindo, dessa forma, a eficiência do uso de irrigação (Bernardo et. al., 2006; Fietz et al. 2005, o método de Hargreaves-Samani foi desenvolvido para regiões semi-áridas, com elevado déficit de saturação.

O método Penman-Monteith, no qual se emprega o conceito de cultura hipotética, é considerado padrão, sendo recomendado por Allen et al. (1998). Embora tenham sido verificados consistência e ótimo desempenho deste método (Jensen et al., 1990), o requerimento de um conjunto amplo de dados como, medidas de saldo de radiação (Rn), fluxo de calor no solo (MJ m${ }^{-2} \mathrm{dia}^{-1}$ ), temperatura $\left({ }^{\circ} \mathrm{C}\right)$, e umidade do ar $(\%)$, pressão atmosférica ( $\mathrm{kPa}$ ), e velocidade do vento a $2 \mathrm{~m}$ de altura $\left(\mathrm{m} . \mathrm{s}^{-1}\right)$, impõe obstáculos à sua difusão, sobretudo aos pequenos produtores para os quais os custos de aquisição de estações meteorológicas automáticas são, ainda, impeditivos (Borges Júnior et al.,2012).

Quando uma base mais ampla de dados meteorológicos não é disponível, faz-se necessário o emprego de métodos mais simples, com menor requerimento de dados. Deve-se observar, contudo, que o desempenho dos vários métodos na determinação da evapotranspiração de referência depende das condições climáticas locais (Jensen et al., 1990; Allen et al., 1998). Dado o empirismo característico de métodos com baixo requerimento de dados de entrada, verificações e calibrações regionais, quando não locais, propiciarão ganhos em termos qualitativos da estimativa da ETo e no estabelecimento do manejo de irrigação, portanto, o uso de métodos empíricos deve ser antecedido por 
avaliações de desempenho regional (Borges Junior, et. al., 2012).

Métodos que utilizam somente a temperatura do ar, umidade relativa do ar e radiação solar para a estimativa da ETo também podem ser empregados pelos produtores para o manejo da irrigação. Conceição \& Marin (2003) observaram um coeficiente de determinação $\left(R^{2}\right)$ igual a 0,84 entre os valores mensais de ETo determinados pelo método de Penman-Monteith-FAO e os valores obtidos pelo método de HargreavesSamani, sob as condições do noroeste paulista, mostrando com isto, que as estimativas da ETo utilizando esse método simplificado tem uma relacao boa com os dados obtidos com o método padrão.

Objetivou-se, neste trabalho, avaliar o desempenho dos métodos Blaney-Criddle, Camargo, Hargreaves-Samani e PriestleyTaylor, de estimativa da evapotranspiração de referência em base diária, para a região de São Luis, MA, com base em comparações com o método padrão Penman-Monteith.

\section{MATERIAL E MÉTODOS}

\section{Base de dados}

Os dados utilizados nas estimativas da ETo, pelos diferentes métodos, foram coletados a partir de uma estação automática Convencional do Instituto Nacional de Meteorologia, INMET, localizada no município de São Luis - MA, na latitude $2^{\circ}$ $31^{\prime \prime} 48^{\prime}$ Sul, longitude $44^{\circ} 12^{\prime \prime} 36^{\prime}$ Oeste e altitude de 50,86 m, empregando-se assim dados meteorológicos em base diária.

O clima é tropical com estação seca (Julho a Dezembro), segundo a classificação climática de Köppen-Geiger: Aw, as temperaturas médias anuais são superiores a $24^{\circ} \mathrm{C}$, enquanto os índices pluviométricos variam entre 1500 e $2500 \mathrm{~mm}$ anuais. Os dados trabalhados foram relativos aos seguintes elementos meteorológicos: temperaturas máxima (Tmax; $\left.{ }^{\circ} \mathrm{C}\right)$, média $\left(\right.$ Tmed; $\left.{ }^{\circ} \mathrm{C}\right)$ e mínima (Tmin; ${ }^{\circ} \mathrm{C}$ ), insolação (h), velocidade do vento média diária $\left(\mathrm{U}_{2} ; \mathrm{m} \mathrm{s}^{-1}\right)$ e umidade relativa do ar média diária (URmed; \%).

Na estação do INMET, a temperatura máxima (Tmax) é obtida a 00:00 UTC (Universal Time, Coordinated); a temperatura mínima (Tmin) é obtida nas 12:00 UTC; a temperatura média (Tmed) (diária) é obtida através da soma da temperatura, nas 12:00 UTC, mais duas vezes a temperatura nas 24:00 UTC, mais a temperatura máxima, mais a temperatura mínima, dividindo-se por cinco; o sensor de velocidade do vento $\left(\mathrm{U}_{2}\right)$ fica a $10 \mathrm{~m}$ de altura; a umidade relativa média diária (URmedia) é obtida pela soma das umidades relativas, às 12:00, 18:00 e duas vezes, nas 24:00 UTC, dividindo-se por quatro; a insolação (n) é medida empregando-se um heliógrafo tipo Campbell-Stokes. A conversão da velocidade do vento para altura de $2 \mathrm{~m}$ foi feita conforme Allen et al. (1998). Os dados foram obtidos de 01 de janeiro de 2012 a 30 de dezembro de 2012.

Na planilha utilizada para a realização das estimativas pelos diferentes métodos, foi realizada uma análise de consistência, visando identificar valores discrepantes, como valores de Tmin superiores a Tmax; valores de Tmedia superiores a Tmax; valores de URmedia superiores a $100 \%$; valores de $U_{2}$ superiores a $8 \mathrm{~m} \mathrm{~s}^{-1}$ e valores de $\mathrm{n}$ superiores a $13 \mathrm{~h}$. Para isto foi realizado um gráfico com cada uma das variáveis em relação ao tempo, assim, com ajuda de uma análise visual dos gráficos complementou-se a verificação de consistência dos dados.

Foi selecionado este ano para estudo por não conter dados faltantes, na série histórica, de insolação, assim como não possuir dados faltantes de Tmax ou Tmin, superior a 10 dias, unicamente foram encontrados dados faltantes de Tmax em alguns dias, que foram corrigidos considerando-se a média dos dias imediatamente anterior e posterior.

Métodos para estimativa da
evapotranspiração de referência


A ETo foi estimada por meio dos métodos de Penman-Monteith FAO 56 (Allen et al., 1998), que é considerado o padrão de referência, Blaney- Criddle, Camargo, Hargreaves-Samani e Priestley-Taylor. O método de Penman-Monteith FAO 56 foi utilizado para testar a estimativa de
ETo obtida com os outros métodos em para realizar a comparação objetivada neste estudo, conforme proposto por Allen et al. (1998).

O método FAO Penman-Monteith é sintetizado na seguinte equação (Allen et al., 1998):

$$
E T_{o}=\frac{0,408 \Delta\left(R_{n}-G\right)+\gamma \frac{900}{T+273} U_{2}\left(e_{s}-e_{a}\right)}{\Delta+\gamma\left(1+0,34 U_{2}\right)}
$$

em que:

$E T o=$ é a evapotranspiração de referência diária (mm dia $\left.{ }^{-1}, \mathrm{~mm} \mathrm{~d}^{-1}\right)$;

$R n=$ é o saldo diário de radiação $\left(\mathrm{MJ} \mathrm{m}^{-2}\right.$ $\left.\mathrm{dia}^{-1}\right)$;

$G=$ é o fluxo de calor no solo diário (MJ $\left.\mathrm{m}^{-2} \mathrm{dia}^{-1}\right)$;

$T$ = é a temperatura do ar a $2 \mathrm{~m}$ de altura $\left({ }^{\circ} \mathrm{C}\right)$;

$U_{2}=$ é a velocidade do vento a $2 \mathrm{~m}$ de altura $\left(\mathrm{m} \mathrm{s}^{-1}\right)$;

$e_{s}=$ é a pressão da saturação do vapor $(\mathrm{kPa})$;

$e_{a}=$ é a pressão atual de vapor $(\mathrm{kPa})$;

$\left(e_{s}-e_{a}\right)=$ déficit de pressão de vapor, (Kpa);

$\Delta$ = é a declividade da curva de pressão de vapor de saturação $\left(\mathrm{kPa}^{\circ} \mathrm{C}^{-1}\right)$; e

$\gamma=$ é a constante psicrométrica $\left(\mathrm{kPa}^{\circ} \mathrm{C}^{-1}\right)$.

O método de Blaney - Criddle - FAO foi desenvolvido relacionando os valores da evapotranspiração mensal com o produto da temperatura média mensal pela percentagem mensal de horas anuais de luz solar, o qual foi modificado pela FAO, incluindo ajustes climáticos locais, sendo assim calculada pela seguinte equação:

$$
E T_{0}=P \cdot\left(0,457 \cdot T_{\text {média }}+8,13\right)
$$

em que,

$E T_{0}$ : evapotranspiração potencial de referência, $\left(\mathrm{mm} \mathrm{dia}^{-1}, \mathrm{~mm} \mathrm{~d}^{-1}\right)$;

$T_{\text {média: }}$ temperatura média diaria, em ${ }^{\circ} \mathrm{C}$; e

$P$ : percentagem diária de horas anuais de luz solar

O método de Camargo é um método empírico, baseado no método de Thornthwaite. Sendo assim, apresenta as mesmas vantagens e restrições desse método. Apesar disso, tem uma vantagem a mais que é não necessitar da temperatura média anual normal. No entanto, considera a irradiância solar extraterrestre (Qo), a qual é fornecida por tabelas.

$$
E T_{0}=0,01 \cdot Q o \cdot \text { Tmed }
$$

em que: $\left(\mathrm{mm} \mathrm{d}^{-1}\right)$;

Qo - irradiância solar extraterrestre

O método de Hargraves - Samani (1985) incorpora, além das temperaturas mínima, média e máxima do ar, a radiação solar recebida em uma superfície horizontal na parte externa da atmosfera. O fator de radiação considerado é função da latitude e do período do ano. A ETo pode ser obtida pela seguinte equação.

$$
E T_{0}=\sqrt{0,0023 \cdot\left(T_{\text {média }}+17,8\right)\left(T_{\max }-T_{\min }\right)} \cdot R a \cdot 0,408
$$

em que, 
$T_{\text {med }}, T_{\max }$ e $T_{\min }-$ Representam, respectivamente, as temperaturas média, máxima e mínima, em ${ }^{\circ} \mathrm{C}$, e

$R a$ - Radiação solar no topo da atmosfera $\left(\mathrm{MJ} \mathrm{m}^{-2} \mathrm{~d}^{-1}\right)$

O método de Priestley-Taylor é um método físico, baseado no método original de Penman, o qual considera que a ETo proveniente do termo aerodinâmico, ou seja, do poder evaporante do ar, é uma porcentagem da ETo condicionada pelo termo energético. Assim, mesmo levando em consideração o balanço de energia, esse método apresenta um componente empírico.

$$
E T_{0}=1,26 \cdot W \cdot(R n-G) / \gamma
$$

em que:

$W=$ fator de ponderação dependente da temperatura do ar (T)

$\mathrm{Rn}=$ saldo diário de radiação

$\mathrm{G}$ = fluxo de calor no solo diário

$\left.{ }^{\circ} \mathrm{C}\right)$

$W=0,407+0,0145 \mathrm{~T}\left(\right.$ para $0{ }^{\circ} \mathrm{C}<\mathrm{T}<16$

$W=0,483+0,01 \mathrm{~T}\left(\right.$ para $\left.\mathrm{T}>16^{\circ} \mathrm{C}\right)$

A análise do desempenho dos métodos avaliados foi realizada por meio da comparação dos valores de evapotranspiração obtidos com os estimados pelo método de Penman-MonteithFAO 56.

\section{Comparação dos métodos}

Como indicadores para comparação de desempenho dos métodos utilizados nas estimativas da ETo frente ao método padrão Penman - Monteith, foram considerados os seguintes parâmetros: erro absoluto médio (EAM), a raiz quadrada do erro quadrado médio (REQM); a raiz quadrada do erro quadrado médio sistemático (REQMs); a raiz quadrada do erro quadrado médio não sistemático (REQMns); o índice de concordância (d); o índice de confiança (c); os coeficientes de correlação (r) e determinação $\left(\mathrm{R}^{2}\right)$. A metodologia adotada para comparação dos resultados foi proposta por Allen et al. (1998), e se fundamenta na estimativa do erro absoluto médio (EAM), calculada pela equação 6. As expressões seguintes foram empregadas
(Willmott, C. J., 1982); Camargo \& Sentelhas; 1997; Willmott, C. J. \& Matsuura, 2005):

$$
\begin{gathered}
E A M=\frac{\sum_{i=1}^{N}|P i-O i|}{N} \\
R E Q M=E Q M^{o, 5}=\left[N_{d}^{-1} \sum_{i=1}^{N}\left(P_{i}-O_{i}\right)^{2}\right]^{\frac{1}{2}} \\
R E Q M s=E^{2} M^{0,5}=\left[N_{d}^{-1} \sum_{i=1}^{N}\left(\hat{P}_{i}-O_{i}\right)^{2}\right]^{\frac{1}{2}}
\end{gathered}
$$

$$
\begin{gathered}
\text { REQMns }=\text { EQMns }^{0,5}=\left[N_{d}^{-1} \sum_{i=1}^{N}\left(\hat{P}_{i}-P_{i}\right)^{2}\right]^{\frac{1}{2}} \\
d=1-\frac{\sum_{i=1}^{N}\left(P_{i}-O_{i}\right)^{2}}{\sum_{i=1}^{N}\left[\left(\left|P_{i}-\bar{O}\right|\right)+\left(\left|O_{i}-\bar{O}\right|\right)\right]^{2}} \\
c=d \cdot r
\end{gathered}
$$

em que:

$P i$ - evapotranspiração de referência obtida pelo método a ser testado, $\mathrm{mm} \mathrm{d}^{-1}$; Oi evapotranspiração de referência obtida pelo método-padrão, $\mathrm{mm} \mathrm{d}^{-1} ; \hat{P}_{i}$ - estimador de Pi com base no modelo de regressão linear, $\mathrm{mm} \mathrm{d}^{-1} ; \bar{O}$ - média dos valores obtidos pelo método-padrão, $\mathrm{mm} \mathrm{d}^{-1} ; N$ - número de observações; e $d$ - índice de concordância ou ajuste.

A exatidão está relacionada com a aproximação dos valores estimados em relação aos valores observados. A aproximação dos valores de ETo estimados pelo método em estudo, em relação aos valores obtidos com o uso do método padrão, foi obtida por meio de um índice designado concordância ou ajuste (d) (Willmott; Ckleson; Davis, 1985), cujos valores variam de zero $(0,0)$, onde não existe 
concordância, a 1, para a concordância perfeita. O índice de aproximação é calculado com a equação 5. Utilizou-se um índice de confiança “c”, ferramenta estatística de comparação proposta por Camargo \& Sentelhas (1997), que serve como indicador de desempenho dos métodos, reunindo os índices de precisão " $\mathrm{r}$ " $\mathrm{e}$ de exatidão “d”, sendo expresso na equação (10).

Os valores desse índice "c" variam de 0,0 para nenhuma concordância a 1,0 para concordância perfeita entre os dados, qualificando os resultados de acordo com a Tabela 1.

Tabela 1 - Classificação do desempenho segundo o índice de confiança "c”.

\begin{tabular}{cc}
\hline Valor de “c” & Desempenho \\
\hline$\geq 0,85$ & Ótimo \\
0,76 a 0,85 & Muito Bom \\
0,66 a 0,75 & Bom \\
0,61 a 0,65 & Mediano \\
0,51 a 0,60 & Sofrível \\
0,41 a 0,50 & Mau \\
$\leq 0,40$ & Péssimo \\
\hline
\end{tabular}

\section{Resultados e Discussão}

Os resultados das comparações em base diária são mostrados na Tabela 2 e na Figura 1 (A, B, C e D). Com base nos resultados do EAM os métodos podem ser ranqueados apresentando a seguinte ordem decrescente: Blaney-Criddle, Camargo, Hargreaves-Samani e Priestley-Taylor, já no parâmetro coeficiente de correlação o método de Priestley-Taylor proporcionou o maior $(0,97)$ e foi, com base no índice de confiança “c” (Tabela 1), o primeiro no ranque.
Observa-se, na Tabela 2, que o método Priestley-Taylor, com base em dados de radiação, proporcionou o menor erro absoluto médio, EAM, dentre todos os avaliados, com valor igual a 0,54. Obtiveram-se valores elevados de coeficiente de correlação $(r=0,97)$ e índice de concordância (d $=0,91$ ) e, consequentemente, de confiança $(c=0,88)$, o que indica desempenho "ótimo" (Tabela 1), sendo seu uso indicado como alternativa ao método Penman-Monteith na ausência de dados de velocidade de vento, para a região de São Luis, MA.

Tabela 2. Comparação dos métodos avaliados com o método padrão FAO Penman-Monteith.

\begin{tabular}{|c|c|c|c|c|c|}
\hline & $\begin{array}{l}\text { Penman- } \\
\text { Monteith }\end{array}$ & $\begin{array}{l}\text { Blaney- } \\
\text { Criddle }\end{array}$ & Camargo & $\begin{array}{c}\text { Hargreaves- } \\
\text { Samani }\end{array}$ & $\begin{array}{l}\text { Priestley- } \\
\text { Taylor }\end{array}$ \\
\hline Média $\left(\mathrm{mm} \mathrm{d}^{-1}\right)$ & 4,29 & 3,7 & 3,94 & 4,18 & 4,78 \\
\hline $\mathrm{Sd}(\mathrm{mm})$ & 0,83 & 0,07 & 0,26 & 0,48 & 0,87 \\
\hline CV $(\%)$ & 19,30 & 1,78 & 6,48 & 11,49 & 18,2 \\
\hline Máximo $\left(\mathrm{mm} \mathrm{d}^{-1}\right)$ & 5,79 & 3,82 & 4,31 & 5,35 & 6,28 \\
\hline Mínimo $\left(\mathrm{mm} \mathrm{d}^{-1}\right)$ & 1,95 & 3,45 & 3,30 & 2,15 & 2,17 \\
\hline $\mathrm{r}$ & & 0,70 & 0,50 & 0,75 & 0,97 \\
\hline EAM (mm) & & 0,98 & 0,81 & 0,58 & 0,54 \\
\hline EQM $\left(\mathrm{mm}^{2}\right)$ & & 0,96 & 0,66 & 0,33 & 0,29 \\
\hline REQM (mm) & & 0,98 & 0,81 & 0,58 & 0,54 \\
\hline EQMs $\left(\mathrm{mm}^{2}\right)$ & & 0,01 & 0,05 & 0,08 & 0,25 \\
\hline REQMs (mm) & & 0,07 & 0,22 & 0,28 & 0,50 \\
\hline EQMns $\left(\mathrm{mm}^{2}\right)$ & & 0,89 & 0,77 & 0,61 & 0,98 \\
\hline REQMns (mm) & & 0,94 & 0,88 & 0,78 & 0,99 \\
\hline $\mathrm{d}$ & & 0,70 & 0,77 & 0,86 & 0,91 \\
\hline C & & 0,49 & 0,38 & 0,64 & 0,88 \\
\hline
\end{tabular}




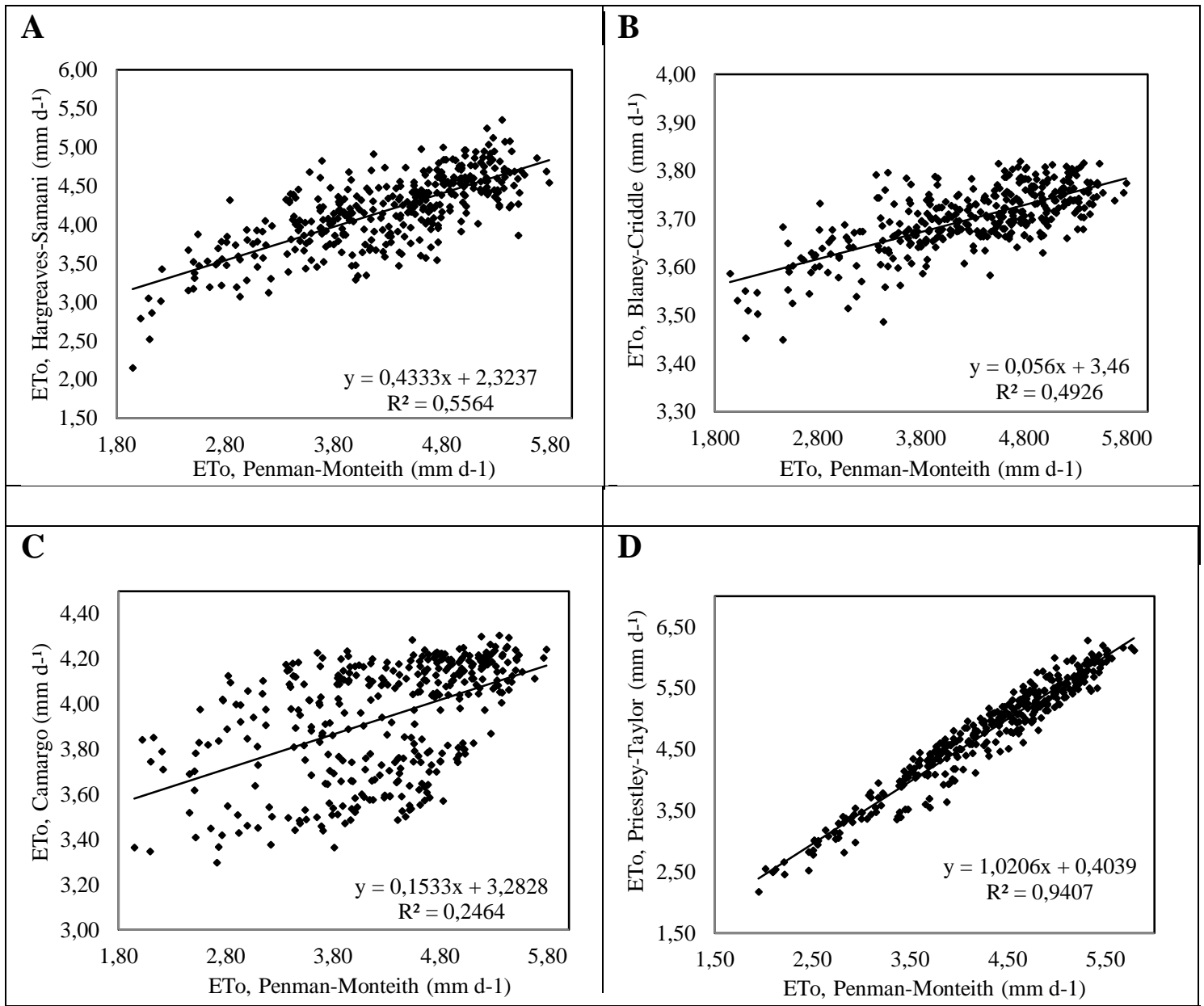

Figura 1. Evapotranspiração de referência diária, ETo, para o ano de 2012, método FAO Penman-Monteith versus métodos avaliados (A: Hargreaves-Samani; B: Blaney-Cridle; C: Camargo; D: Priestley-Taylor), comparados por regressão linear.

Embora que o método Priestley-Taylor apresente a melhor exatidão $(\mathrm{d}=0,91)$, nota-se na Figura 1-D, que a maioria dos dados estimados, em relação ao método padrão de Penman-Monteith, estão abaixo da linha da regressão linear o que determina que o método superestima os valores da ETo, sendo por este motivo que o valor do EQMs seja o valor mais elevado dos métodos avaliados.

Em estudo aplicado no estado da Geórgia (EUA) e comparando os métodos PriestleyTaylor e FAO Penman-Monteith, Suleiman e Hoogenboom (2007) encontraram valores de d variando de 0,95 a 0,99. Fietz \& Fisch (2009) verificaram desempenho "muito bom" deste método, com c $=0,78$, com tendência de superestimativa.

Dos métodos que utilizam apenas dados de temperatura para estimar o valor da ETo, o método de Hargreaves-Samani foi o que apresentou menor EAM, sendo de 0,58, com um valor do coeficiente de correlação $r=0,75$ e índice de concordância $d=0,86$ o que resultaria, por conseguinte, um índice de confiança $\mathrm{c}=0,64$, permitindo, assim, classificar o desempenho da estimativa da ETo por este método como "mediano" (Tabela 1). Já o método de Blaney-Criddle apresentou o maior valor de $\mathrm{EAM}=0,98$, com valores no coeficiente $r=0,70$, no $d=0,70$ e $\mathrm{c}=0,49$, o que permite classificar o desempenho de este método como "Mau" (Tabela 1). Igualmente, realizando este analise, o método de Camargo foi o que mostrou o pior desempenho, em que os valores obtidos não apresentaram bom ajuste em relação ao método de PenmanMonteith, ocorrendo dispersão dos dados, com valores do $\mathrm{r}=0,50$ e $\mathrm{d}=0,77$ e $\mathrm{c}=0,38$, permitindo assim ser classificado, segundo Tabela 1, como "Péssimo”. 
Em trabalho realizado por Vallory, et. al., 2016, concluem, de igual forma, que o método do Camargo, para a estimativa da evapotranspiração referência em escalas diárias, não se pode recomendar em condições climáticas de verão, que são as condições climáticas normalmente encontradas na localidade de estudo.

Realizando a análise da relação entre as diferentes variáveis climáticas, disponíveis na estação meteorológica, e a estimativa da ETo pelo método de Penman-Monteith, como mostrado na Figura 2, é possível observar que a variável que mais ter correlação com a ETo, neste método avaliado, é a insolação, com $r=$ 0,89 .

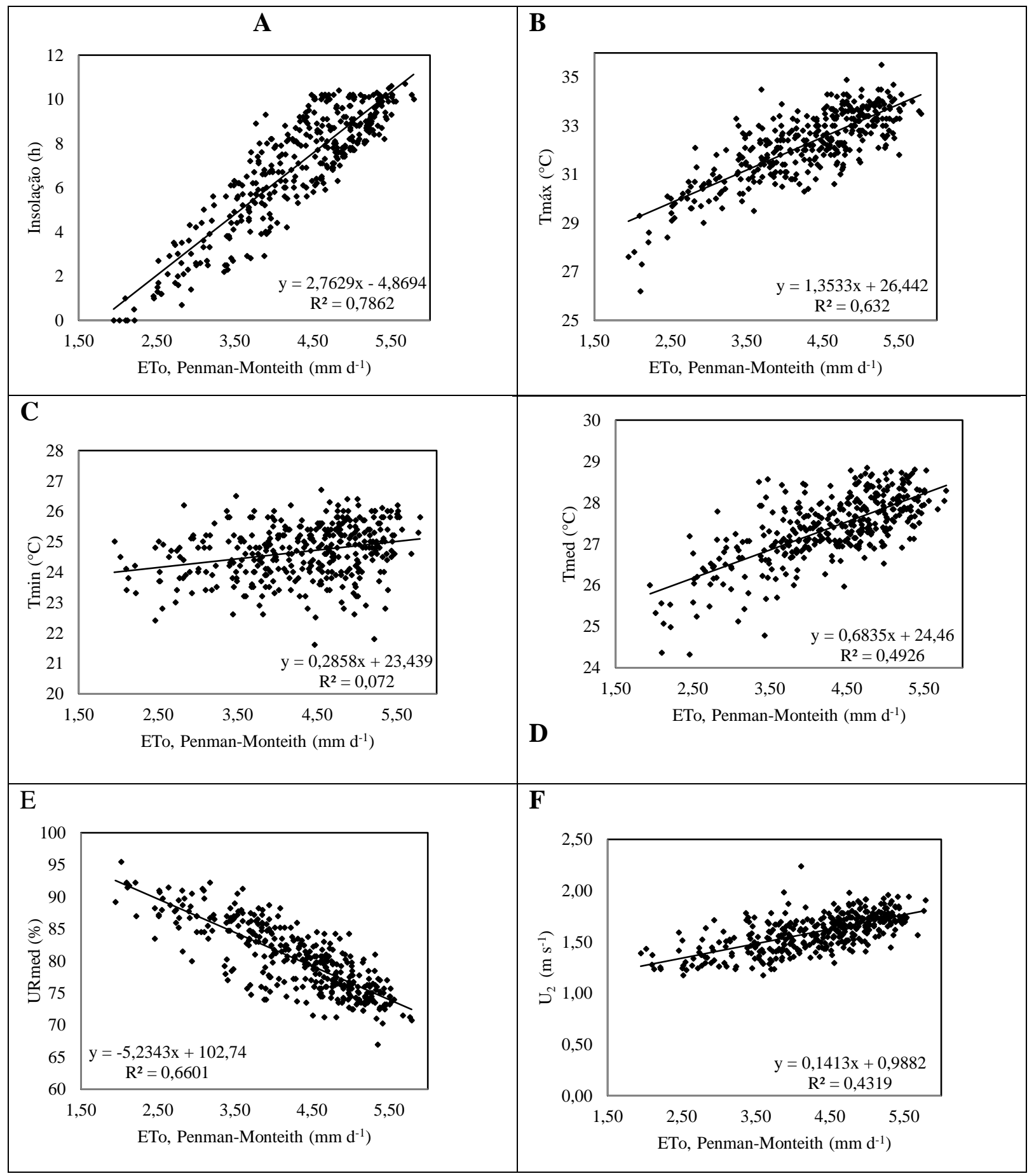

Figura 2. Relações entre os valores diários da evapotranspiração de referência calculado pelo método FAO Penman-Monteith com as diferentes variáveis climáticas disponíveis. 
De acordo com o trabalho de Amorim et al. (2007), que teve como objetivos determinar os efeitos direto e indireto dos elementos meteorológicos no processo de ETo estimadas pelo modelo de Penman-Monteith (FAO) e Tanque Classe A, na região agrícola de Mossoró, a insolação foi o elemento do clima de maior contribuição para ETo, o que corrobora o mostrado na Figura 2-A.

Seguidamente, em ordem de correlação das variáveis, a URmed foi a que apresentou o seguinte valor de $r$ sendo de 0,81 , seguida da Tmax com $r=0,75$, a Tmed com $r=0,70$, a Vv com $r=0,66$ e a variável que apresentou a menor correlação com a ETo foi a Tmin = 0,27.

\section{CONCLUSÕES}

Dentre os métodos avaliados verificouse que o melhor desempenho foi obtido com o método de Priestley-Taylor, com base nos valores do erro médio absoluto, EAM, e no coeficiente de confiança, $\mathrm{C}$, embora os valores estimados por este método em relação ao padrão foram superestimados.

Em relação aos métodos com requerimento apenas de dados de temperatura, o de Hargreaves-Samani calibrado foi o que apresentou o melhor desempenho.

\section{REFERÊNCIAS BIBLIOGRÁFICAS}

ALLEN, R. G. et al. Crop evapotranspiration: guidelines for computing crop water requirements. Roma: FAO, 300p. (Irrigation and Drainage, n.56). 1998.

BERNARDO, S. SOARES, A. A. MANTOVANI, E.C. Manual de irrigação. Viçosa: Ed. UFV, 2006. 625p.

BORGES JÚNIOR, J. C. F.; ANJOS, R. J.; SILVA, T. J. A.; LIMA, J. R. S.; ANDRADE, C. L. T. Métodos de estimativa da evapotranspiração de referência diária para a microrregião de Garanhuns, PE. Revista Brasileira de Engenharia Agrícola e
Ambiental, Campina Grande, v. 16, n. 4, p. 380-390, 2012

AMORIM, C.A.; Sediyama, G.C.; Sobrinho, J.E. \& Justino, F.B. 2007. Análise dos efeitos diretos e indiretos das variáveis meteorológicas no processo de evapotranspiração. In: CONGRESSO BRASILEIRO DE AGROMETEOROLOGIA, XV, Aracaju, 2007. Trabalho completo, Aracaju, SBA, CDROOM.

CAMARGO, A. P.; SENTELHAS, P. C. Avaliação do desempenho de diferentes métodos de estimativa da evapotranspiração potencial no estado de São Paulo, Brasil. Revista Brasileira de Agrometeorologia, v.5, p.89-97, 1997.

CONCEIÇÃO, M.A.F.; MARIN, Estimativa da evapotranspiração de referência com base na temperatura do ar para as condições do Baixo Rio Grande, SP. Revista Brasileira de Agrometeorologia, v.11, n.2, p.229- 236, 2003.

FERRAZ, R. C.; Estimativa da evapotranspiração de referência utilizando redes neurais artificiais para o Estado do Rio Grande do Sul. Revista Tecnológica. v.23, p.25-31, 2014

FIETZ, C. R.; FISCH, G. F. Avaliação de modelos de estimativa do saldo de radiação e do método de Priestley-Taylor para a região de Dourados, MS. Revista Brasileira de Engenharia Agrícola e Ambiental, v.13, p.449-53, 2009.

FIETZ, C.R.; SILVA, F.C; URCHEI, M.A. 2005. Estimativa da evapotranspiração de referência diária para a região de Dourados, MS. Revista Brasileira de Agrometeorologia, 13(2): 250-255.

JENSEN, M. E.; BURMAN, R. D.; ALLEN, R. G. Evapotranspiration and irrigation water requirements. American Society of Civil Engineers, New York, 1990. 332p. 
OLIVEIRA, M.A.A.; CARVALHO, D.F. Estimativa da evapotranspiração de referência e da demanda suplementar de irrigação para o milho (Zea mays L.) em Seropédica e Campos, Estado do Rio de Janeiro. Revista Brasileira de Engenharia Agrícola e Ambiental, 2(2): 132-135. 1998.

PRIESTLEY, C.H.B; TAYLOR, R.J. On the assessment of surface heart flux and evaporation using large-scale parameters. Monthly Weather Review, v.100, n.2, p.8192, Fev. 1972.

SILVA, A.A.G.; ANGELOCCI, L.R.; NOGUEIRA, L.C.; ANDRADE, C.L.T. Avaliação da eficiência de métodos de estimativa da evapotranspiração de referência (ETo). In: CONGRESSO BRASILEIRO DE ENGENHARIA AGRÍCOLA, 22., 1993, Ilhéus. Anais... Ilhéus: Sociedade Brasileira de Engenharia Agrícola, 1993. p.2465-78.

SULEIMAN, A. A.; HOOGENBOOM, G. Comparison of Priestley-Taylor and FAO-56 Penman-Monteith for Daily Reference Evapotranspiration Estimation in Georgia.
Journal of Irrigation and Drainage Engineering, v. 133, p.175-82, 2007.

VALLORY, N. D.; DOHLER, R. E.; CECÍLIO, R. A.; ZANETTI, S. S. Métodos empíricos para estimativa da evapotranspiração de referência no Estado do Rio de Janeiro. Revista Brasileira de Agricultura Irrigada. v.10, n.2, p. 576 - 585, 2016.

WILLMOTT, C. J.; CKLESON, S. G.; DAVIS, R. E. Statistics for evaluation and comparisons of models. Journal of Geophysical Research, Ottawa, v.90, n.C5. p.8995-9005, 1985.

WILLMOTT, C. J. Some comments on the evaluation of model performance. Bulletin American Meteorological Society, v.30, p.1309-1310, 1982.

WILLMOTT, C. J.; MATSUURA, K. Advantages of the mean absolute error (MAE) over the root mean square error (RMSE) in assessing average model performance. Climate Research, v.30, p.79-82, 2005. 